\title{
Compact circuits for combined AES encryption/decryption
}

\author{
Banik, Subhadeep; Bogdanov, Andrey; Regazzoni, Francesco
}

Published in:

Journal of Cryptographic Engineering

Link to article, DOI:

$10.1007 / \mathrm{s} 13389-017-0176-3$

Publication date:

2019

Document Version

Peer reviewed version

Link back to DTU Orbit

Citation (APA):

Banik, S., Bogdanov, A., \& Regazzoni, F. (2019). Compact circuits for combined AES encryption/decryption. Journal of Cryptographic Engineering, 9(1), 69-83. https://doi.org/10.1007/s13389-017-0176-3

\section{General rights}

Copyright and moral rights for the publications made accessible in the public portal are retained by the authors and/or other copyright owners and it is a condition of accessing publications that users recognise and abide by the legal requirements associated with these rights.

- Users may download and print one copy of any publication from the public portal for the purpose of private study or research.

- You may not further distribute the material or use it for any profit-making activity or commercial gain

- You may freely distribute the URL identifying the publication in the public portal

If you believe that this document breaches copyright please contact us providing details, and we will remove access to the work immediately and investigate your claim 


\title{
Compact Circuits for Combined AES Encryption/Decryption
}

\author{
Subhadeep Banik • Andrey Bogdanov • Francesco Regazzoni
}

Received: date / Accepted: date

\begin{abstract}
The implementation of the AES encryption core by Moradi et al. at Eurocrypt 2011 is one of the smallest in terms of gate area. The circuit takes around 2400 gates and operates on an 8 bit datapath. However this is an encryption only core and unable to cater to block cipher modes like CBC and ELmD that require access to both the AES encryption and decryption modules. In this paper we look to investigate whether the basic circuit of Moradi et al. can be tweaked to provide dual functionality of encryption and decryption (ENC/DEC) while keeping the hardware overhead as low as possible. We report two constructions of the AES circuit. The first is an 8-bit serialized implementation that provides the functionality of both encryption and decryption and occupies around $2605 \mathrm{GE}$ with a latency of 226 cycles. This is a substantial improvement over the next smallest AES ENC/DEC circuit (Grain of Sand) by Feldhofer et al. which takes around 3400 gates but has a latency of over 1000 cycles for both the encryption and decryption cycles.

In the second part, we optimize the above architecture to provide the dual encryption/decryption functionality in only $2227 \mathrm{GE}$ and latency of $246 / 326$ cycles for the encryption and decryption operations respectively. We take advantage of clock gating techniques
\end{abstract}

Sections 1, 2 and 3 appeared in the article "Atomic-AES: A Compact Implementation of the AES Encryption/Decryption core" accepted in Indocrypt 2016, LNCS 10095, pp. 173-190.

Subhadeep Banik

LASEC, École Polytechnique Fédérale de Lausanne, Switzerland, E-mail: subhadeep.banik@epfl.ch

Andrey Bogdanov

DTU Compute, Technical University of Denmark, Lyngby

E-mail: anbog@dtu.dk

Francesco Regazzoni

ALARI, University of Lugano. E-mail: regazzoni@alari.ch to achieve Shiftrow and Inverse Shiftrow operations in 3 cycles instead of 1 . This helps us replace many of the scan flip-flops in the design with ordinary flip-flops. Furthermore we take advantage of the fact that the Inverse Mixcolumn matrix in AES is the cube of the Forward Mixcolumn matrix. Thus by executing the Forward Mixcolumn operation three times over the state, one can achieve the functionality of Inverse Mixcolumn. This saves some more gate area as one is no longer required to have a combined implementation of the Forward and Inverse Mixcolumn circuit.

Keywords AES 128 - Serialized Implementation

\section{Introduction}

There has been extensive research into the construction of compact implementations of lightweight block ciphers. This line of research has essentially evolved along two different lines. The first aims to construct proprietary lightweight block ciphers by optimizing one or several parameters in the design spectrum, as has been evidenced by numerous such designs proposed in the past few years: HIGHT [21, KATAN [1], Klein [18, LED [19, Noekeon [13, Present 7], Piccolo 27, Prince [8], Simon/Speck [6] and TWINE [28]. The second aims at attempting to implement standardized ciphers like AES 128 [14] in a lightweight fashion.

There have been several lightweight implementations of AES proposed in literature. Some results like [20] and [10] aim for compact implementations in ASIC and FPGA platforms respectively (however the work in 20] is for an encryption only core). The works in 22] and 29] aim at lowering critical path and increasing throughput. And the works in 3 and 5 aim to 
implement circuits with low energy consumption per encryption operation.

For compact implementations of the dual encryption/decryption circuit, the following results are known. In [26], the authors propose a 32-bit serial architecture with optimized tower field implementation of the S-box and a combinatorial optimization of the Mixcolumn circuit. The size of this implementation was around $5400 \mathrm{GE}$ (gate equivalents, i.e. area occupied by an equivalent number of 2 -input NAND gates). The "Grain of Sand" implementation [17] by Feldhofer et al. constructs an 8-bit serialized architecture with circuit size of around 3400 GE but a latency of over 1000 cycles for both encryption and decryption. Very recently in [23], the authors report an 8-bit serial implementation that takes 1947/2090 GE for the encryption/decryption circuits respectively. This implementation makes use of intermediate register files that can be synthesized in the ASIC flow using memory compilers.

The implementation by Moradi et al. in [25] with size equal to $2400 \mathrm{GE}$ and encryption latency of 226 cycles is one of the smallest known architectures for AES. The design combines 8-bit and 32-bit serial datapaths in a manner that achieves a surprisingly compact implementation. The design uses scan flip-flops for constructing the registers for the state update and key schedule. A scan flip-flop occupies 1 GE less area than the combination of an ordinary flip-flop and a 2:1 multiplexer, while offering the same functionality. Thus this trick saves 1 GE per flip-flop used. This implementation also uses a 32 bit Mixcolumn circuit instead of the 8-bit serialized structure of [17], because the authors argue that any savings in area achieved by an 8-bit serial circuit is offset by the additional registers required to store its output. Finally since each round function in this circuit is implemented in 21 cycles, the control system is made using a 21 cycle LFSR that generates all timing signals accordingly. However this circuit is an encryption-only core, and therefore cannot be used to implement modes like CBC [16], COPA [2], ELmD [15], POET [1] that require access to both AES encryption and decryption functionalities. Therefore area-wise the three smallest known circuits that perform the dual functionalities of both encryption and decryption are
A. Grain of Sand implementation [17] at $3400 \mathrm{GE}$
B. 8-bit serial implementation in 23] at $4037 \mathrm{GE}$
C. 32-bit serial implementation in 26] at $5400 \mathrm{GE}$.

Moreover the Grain of Sand implementation has a latency of over 1000 cycles for both the encryption and decryption operations and so for efficient lightweight implementation of all modes that require access to both AES encryption and decryption it is critical to have an architecture that is both lightweight and incurs minimal latency.

\subsection{Contribution and Organization}

In this paper we present two circuits. The first, AtomicAES, is an 8-bit serial architecture that performs the dual functionality of encryption and decryption, and has a circuit size of around 2605 GE when synthesized with the standard cell library of the STM 90nm CMOS logic process. The circuit has a latency of 226 cycles for both encryption and decryption operations. The circuit is closely related to the 8-bit encryption only serial architecture presented in [25, and in fact the architecture has the following additional logic components over the basic circuit proposed by Moradi et al.

1. 2 additional 8-bit multiplexers added to the state datapath,

2. 3 additional 8-bit xor gates in the key datapath,

3. 24 additional and gates in the key datapath,

4. 1 additional 8-bit multiplexer, 1 additional 8-bit xor gate, 16 additional and gates during state-key addition,

5. Other additional logic required to implement
a. S-box and its inverse,
b. Mixcolumn and its inverse,
c. Round constants and their inverses.

Thereafter, we propose the Atomic-AES v2.0 architecture that at $2227 \mathrm{GE}$, occupies around $400 \mathrm{GE}$ less using the same standard cell library. The architecture has encryption/decryption latency of 246/326 cycles. Each encryption round is computed in 23 cycles, each decryption round takes 31 cycles. The savings in area comes from principally two avenues:

1. The Shiftrow/Inverse Shiftrow operations are performed over three cycles rather than 1 . This reduces the number of 2 input flip-flops required in the design. So it helps the designer replace a lot of the scan flip-flops in the design with ordinary flip-flops. A scan flip-flop usually occupies 1 GE more than an ordinary flip-flop. Hence this trick, on average, saves $1 \mathrm{GE}$ of area per bit of storage.

2. Additionally the Inverse Mixcolumn matrix used in AES is the cube of the Forward Mixcolumn matrix. This implies that executing the Mixcolumn operation 3 times over the state achieves the functionality of Inverse Mixcolumn. Thus the designer no longer needs a combined implementation of the Forward and Inverse Mixcolumn Circuit.

The paper is organized in the following manner. Section 2 gives some background and description of the architecture presented in 25. This would be beneficial for 
the self-sufficiency and better understanding of this paper. Section 3, describes the architecture and functioning of Atomic-AES in details, and highlights some issues related to its implementation. Section 4 describes the architecture and functioning of Atomic-AES v2.0. Section 5 tabulates all implementation results and compares it with previous architectures present in literature. Section 6 concludes the paper.

\section{Background and Preliminaries}

In Figure 1, a pictorial description of the architecture in 25] is given. As can be seen the basic elements of storage are the 16 byte sized registers made of scan flip-flops in the state and key path respectively, used to store the intermediate states and roundkeys. Each round function is calculated in 21 cycles and so it is important to understand how the data is maneuvered through the registers during this period. An important point to note is that this particular architecture interprets the AES input vectors in a row major fashion i.e. the first four bytes are placed in the first row, the second four bytes in the second row so on. Most AES implementations use a column major ordering. If column major ordering is needed, 20 additional 8-bit multiplexers are required. However since the reordering costs are marginal, we assume that it is performed offline by some processor or microcontroller that communicates with the hardware circuit.

Let us label the 21 cycles per round by the integers 0 to 20 . The encryption process starts with the addition of the whitening key and the S-box computation of the first round function. In order to do so the finite state machine (FSM) generating the timing signals is initialized to cycle number 5 . So in cycles numbered 5 to 20 (i.e. the very first 16 cycles) the following transformations take place:

Cycles 5 to 20: The 8 bit chunks of plaintext and key are respectively filtered out of the main state and key multiplexers respectively. They are xored, and the resultant signal fed to the S-box. The output of the S-box is fed to the bottom most multiplexer in the state path (marked by $S B_{I N}$ ), from where it is shifted serially forward in the next round. Effectively, after the cycle 20 is completed, the state registers would store the value $S(P T \oplus K)$, where $S(\cdot)$ denotes the bytewise application of the AES S-box function. In the same period the 8 bit chunk of the Key is input to key register marked " 33 ", from where it is serially forwarded in the next round, much like in the state register. Therefore, at the end of cycle 20, the Key registers hold the value of the initial whitening key.

After this the cycle counter is automatically reset to 0 , and each 21 cycle round function is executed 10 times, thus accounting for a total latency of $16+21 * 10=226$. During this period the order of operations is as follows:

Shiftrow $\rightarrow$ Mixcolumn $\rightarrow$ Add roundkey + S-box ${ }^{*}$

where S-box* denotes the substitution layer of the subsequent round function. To clarify, let us see the cyclewise description of the data movement:

Cycle 0: This cycle is used for the Shiftrow operation. Since each 8-bit register in the state and key paths are constructed using scan flip-flops, they have two input data ports which they filter depending on a select signal. As can be seen in Figure 1, the state registers are connected to facilitate the Shiftrow operation during cycle 0 . The key register is "frozen" in this cycle and so no data movement takes place. 1

Cycles 1 to 4: The Mixcolumn operation is performed during these 4 cycles. The Mixcolumn circuit used in this architecture is a $\{0,1\}^{32} \rightarrow\{0,1\}^{32}$ logic block, and so data from leftmost column (registers marked $00,10,20,30$ ) of the state is fed as input to the Mixcolumn circuit. In the subsequent cycle the Mixcolumn output is driven into the rightmost column (registers marked 03,13,23,33). This operation carried out over 4 cycles computes the Mixcolumn over the entire state. Note that this operation is bypassed in the 10th encryption round as the Mixcolumn function is omitted in the final round.

During this period, the non-linear function of the Keyschedule operation is computed in the Key registers. Recall that the non linear operation in the AES Keyschedule is given as

$F\left(K_{3}\right)=S\left(K_{3} \lll 8\right) \oplus R C O N_{i}$,

where $K_{3}$ denotes the third column of the current roundkey, « denotes the left rotate operation and $R C O N_{i}$ is the $i^{t h}$ round constant (note that the round constant is added to the most significant byte of $\left.S\left(K_{3} \lll 8\right)\right) .\left(K_{3} \lll 8\right)$ is a 32 bit value and so $S\left(K_{3} \lll 8\right)$ implies the S-box function applied to each of the 4 bytes of the input. In order to implement the rotation operation, the data is taken from the output of the key register marked " 13 " and fed to the S-box. Although the architecture uses only

1 One way to achieve this is to use a gated clock which does not present a leading edge during the shiftrow period. 


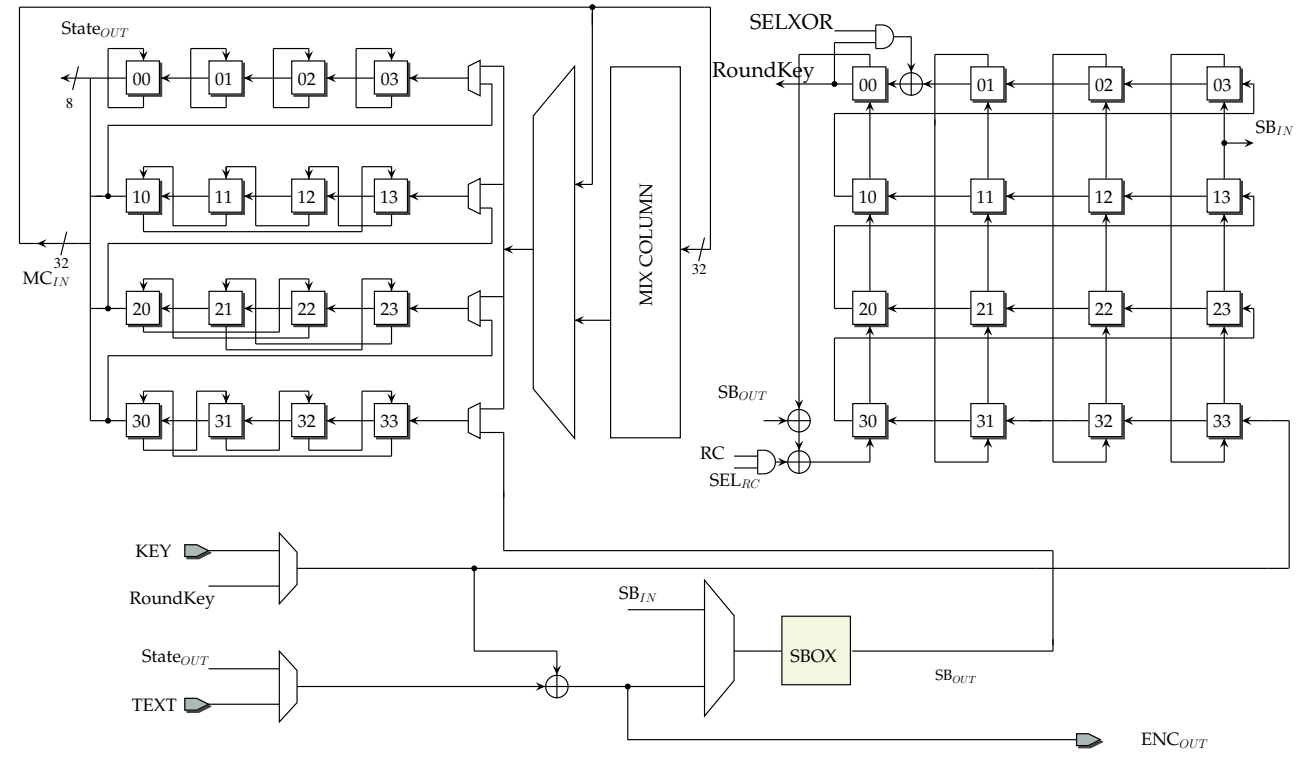

Fig. 1: The 8-bit serial architecture in 25

one S-box, in cycles 1 to 4 , the state path operations do not use the S-box circuit and so the key path S-box operations can be done in this period. The S-box output is xored to the output of the register " 00 " and the round constant and, in the next cycle is driven into the register marked "30". Note that since there is "vertical" movement of data in the key registers in this period, at the end of cycle 4 , the four columns of the key register store the values $K_{0} \oplus F\left(K_{3}\right), K_{1}, K_{2}, K_{3}$ respectively, where $K_{i}$ denotes the $i^{\text {th }}$ column of the current roundkey.

Cycles 5 to 20: The bytes of state and roundkey are respectively taken out of the registers marked " 00 " of both the state and key paths and xored together and fed to the S-box. The output of the S-box is again driven into the bottom most state register "33" and serially shifted forward in the subsequent rounds. This sequence of operations is exactly same as the ones performed in the very first 16 cycles, with the only exception that an intermediate state and roundkey chunks are xored instead of the raw plaintext and key.

The operations in the Key register are a little more interesting during this period. Note that in order to perform roundkey addition during these cycles, the data emanating from key register " 00 " needs to be equal to the current roundkey. However we have seen that at the end of cycle 4 the columns of the key registers hold the value $K_{0} \oplus F\left(K_{3}\right), K_{1}, K_{2}, K_{3}$. Note that if $K_{0}, K_{1}, K_{2}, K_{3}$ and $L_{0}, L_{1}, L_{2}, L_{3}$ denote the 4 columns of the current and next roundkey then we have

$L_{0}=K_{0} \oplus F\left(K_{3}\right), \quad L_{1}=K_{1} \oplus L_{0}, \quad L_{2}=K_{2} \oplus L_{1}$, $L_{3}=K_{3} \oplus L_{2}$.

Thus at the end of cycle 4 , only the $0^{\text {th }}$ column holds the correct next roundkey $L_{0}$. The problem is solved by having an extra xor gate taking inputs from the registers " $00 "$ and "01" and output feeding into "00". Since the movement of data is switched to "horizontal", this helps to perform on the fly addition as the key chunks are driven out of the " 00 " register. The addition is however not executed at cycles $8,12,16,20$ by zeroing the SELXOR signal because as previously noted, the $0^{t h}$ column already has the required roundkey. Also after the roundkey addition, each 8-bit key is circularly shifted back into the key registers through register " 33 " in order to facilitate the operations in the next round function.

The $i^{\text {th }}$ round in this architecture computes the Substitution layer for the $(i+1)^{t h}$ AES encryption round. This being so, in the tenth and final encryption round the only operations that need be performed are Shiftrows and the final roundkey addition. Thus in the tenth round, the Mixcolumn operation is bypassed in cycles 1-4 and the output ciphertext is available just after the roundkey addition from cycles 5 through 20 .

\section{Atomic-AES: Architecture and Dataflow}

We will now present a full description of the proposed architecture for Atomic-AES which provides dual func- 
tionalities for encryption and decryption. A diagram for the proposed architecture is presented in Figure 2 . The architecture builds on the basic circuit in [25], and so the functioning of the circuit during encryption is exactly as described in Section 2 .

\subsection{Issues with the Decryption Circuit}

In order to accommodate decryption operation in the basic circuit of [25], there are some principal difficulties. We will list them one by one:

1. Shiftrow/Inverse Shiftrow: In the Shiftrow operation, the data in the $i^{\text {th }}$ row is left-rotated by $i$ bytes $(0 \leq i \leq 3)$. Hence the Inverse Shiftrow operation would require the $i$-byte right-rotation of the $i^{t h}$ row data. However, in order to accommodate Inverse Shiftrow, and Forward Shiftrow simultaneously we would potentially require another multiplexer at the input of each 8-bit state register.

2. Forward/Inverse Keyschedule: The Keyschedule used in AES has a non-linear shift register like structure, and it is obvious that the key register structure in 25] was explicitly constructed to accommodate its unique mathematical structure, and at the same time produce the current roundkey in an 8-bit serial fashion. It is not immediately clear how the Inverse Keyschedule could be arranged in such a circuit without increasing the circuit size significantly. Also we must note that for decryption, the inverse cipher key (i.e. the final round encryption key) must be made available, because all roundkeys in the decryption circuit are calculated in the reverse order.

3. Sequence of operations during Decryption: The circuit in 25] requires 21 cycles to complete a round function, with the order of operations being: Shiftrows, Mixcolumn followed by Add roundkey and the S-box layer of the following round. It is however not clear what order of operations would achieve the most efficient circuit for decryption. If one chooses to have roughly the same order of operations i.e. Inverse Shiftrows, Inverse Mixcolumn followed by Add roundkey and Inverse S-box, then as per the specification of the Decryption function, we would require the Inverse Mixcolumn of the roundkey as well (as described in [26]). This would most likely require additional cycles to compute the Inverse Mixcolumn of the roundkey and thus increase the latency.

\subsection{Inverse Shiftrow}

An efficient Encryption/Decryption circuit would need to address all the above issues judiciously. To begin with let us address the issue of Shiftrow/Inverse Shiftrow. We make the following observations before proceeding:

Observation 1: For the $0^{\text {th }}$ and the $2^{\text {nd }}$ rows of the AES state, Shiftrow and Inverse Shiftrow bring about the same transformation.

Observation 2: For the $1^{\text {st }}$ and the $3^{\text {rd }}$ rows of the AES state, Shiftrow and Inverse Shiftrow bring about opposite transformations. Which is to say, that the Shiftrow operation on the $1^{\text {st }}$ row brings about the same transformation as the Inverse Shiftrow on the $3^{\text {rd }}$ row and vice versa.

A careful examination of the architecture in [25] reveals that each 8-bit register (constructed with scan flip-flops) accepts two inputs (see Figure 1): one from the register immediately to its right (the rightmost register accepts its input from the leftmost register of the row below it), this connection is to facilitate the serial loading and unloading of the bytes in the state during cycles 5 to 20 . The other input facilitates the transfer of data during they Shiftrow cycle. However, for the first three registers of the $1^{\text {st }}$ row (i.e. " $10 "$, " 11 " and "12") the two inputs are actually the same. So in order to accommodate the Inverse Shiftrow, the second input connection of these three registers can be rewired (see Figure 2) just like in the third row (since the Inverse Shiftrow of the first and Forward Shiftrow of the third row are actually identical transformations). For the last register of this row i.e. "13", an extra multiplexer with input from " 10 " is required. And that solves the problem for the first row.

For the $3^{r d}$ row, the situation is even more straightforward. One of the direct results of Observation 2, is that the first input connection for the registers " 30 ", "31" and "32" (used primarily for serial loading of data) can be used for the dual purpose of performing Inverse Shiftrow. This being the case there is no need for rewiring the inputs. However just as in the $1^{\text {st }}$ row, for register "33", an extra multiplexer with input from register "30" is required. Also as per Observation 1, no change in wiring or logic is required in the $0^{\text {th }}$ and $2^{\text {nd }}$ rows. In Table 2, we summarize the input connections for the first and third row state registers during the various operation stages. For example during serial loading/unloading, register ' 13 ' accepts data coming from register ' 20 ', whereas it takes data from ' 10 ' /' 12 ' during Shiftrow/Inverse Shiftrow respectively. As seen in Figure 2 the register ' 33 ' takes data from the $\mathrm{DEC}_{O U T}$ pin during the serial loading phase (i.e. cycles 5 to 20). 


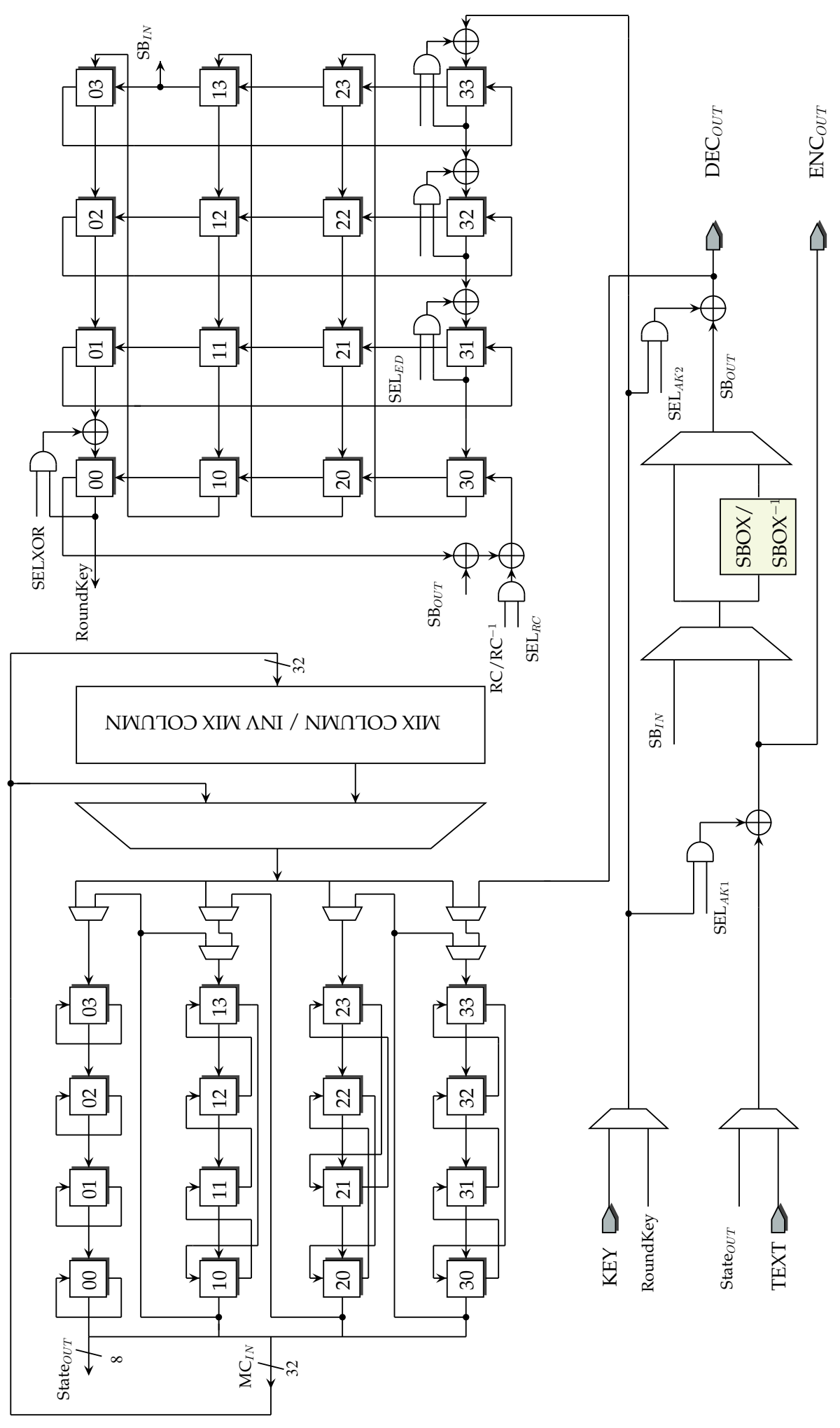

Fig. 2: The AES 8 bit Encryption/Decryption architecture for Atomic-AES 


\begin{tabular}{|c|c|c|c|c|c|c|c|c|c|}
\hline \# & Register & SL & SR & ISR & \# & Register & SL & SR & ISR \\
\hline & \multicolumn{4}{|l|}{ Row 1} & & \multicolumn{4}{|l|}{ Row 3 } \\
\hline 1 & 10 & 11 & 11 & 13 & 1 & 30 & 31 & 33 & 31 \\
\hline 2 & 11 & 12 & 12 & 10 & 2 & 31 & 32 & 30 & 32 \\
\hline 3 & 12 & 13 & 13 & 11 & 3 & 32 & 33 & 31 & 33 \\
\hline 4 & 13 & 20 & 10 & 12 & 4 & 33 & $\mathrm{DEC}_{O U T}$ & 32 & 30 \\
\hline
\end{tabular}

Table 1: Input connections to the 1st and 3rd row state registers during various stages of the operation. (SL: Serial Loading, SR: Shiftrow, ISR: Inverse Shiftrow)

\subsection{Inverse Keyschedule}

To recall, if $K_{0}, K_{1}, K_{2}, K_{3}$ and $L_{0}, L_{1}, L_{2}, L_{3}$ denote the 4 columns of the current and next roundkey then we have

$L_{0}=K_{0} \oplus F\left(K_{3}\right), \quad L_{1}=K_{1} \oplus L_{0}, \quad L_{2}=K_{2} \oplus L_{1}$,

$L_{3}=K_{3} \oplus L_{2}$.

During decryption, the roundkeys are generated in reverse order and so in the context of decryption, $\mathbf{L}=$ $L_{0}, L_{1}, L_{2}, L_{3}$ is essentially the current roundkey and $\mathbf{K}=K_{0}, K_{1}, K_{2}, K_{3}$ is the key to be generated in the subsequent round. So we rewrite the above relation as

$$
\begin{aligned}
& K_{3}=L_{2} \oplus L_{3} \\
& K_{2}=L_{1} \oplus L_{2} \\
& K_{1}=L_{0} \oplus L_{1} \\
& K_{0}=F\left(K_{3}\right) \oplus L_{0}=F\left(L_{2} \oplus L_{3}\right) \oplus L_{0}
\end{aligned}
$$

So in order to have an Encryption/Decryption circuit we need an architecture around the key registers that can both (a) generate $\mathbf{L}$ given $\mathbf{K}$ as input and (b) generate $\mathbf{K}$ given $\mathbf{L}$ as input. The basic architecture in [25] all ready achieves (a) and so we need accommodate (b) i.e. the roundkey generation mechanism during decryption. We offer the following solution. Place three 8-bit xor gates in the $3^{\text {rd }}$ row of Key registers in the following way (refer to Figure 2).

1. For $1 \leq i \leq 2$, the xor gate takes inputs from the key registers " $3 i$ " and " $3(i+1)$ " and feeds its output into register " $3 i$ ".

2 . The third xor gate takes inputs from the registers " 33 " and the current roundkey byte and feeds its output into register " 33 ".

3. For each of these xor gates, the input coming from register " $3 i$ " is anded with a $\mathrm{SEL}_{E D}$ signal. This is done so that serial loading and unloading can be done when required by simply zeroing the $\mathrm{SEL}_{E D}$ signal.

To understand how the Inverse Keyschedule works, let us look at the flow of data in cycles 5 to 20 . For the purpose of simplification let $L_{0 i}, L_{1 i}, L_{2 i}, L_{3 i}$ denote the 4 key bytes in the column $L_{i}$, and similarly let $K_{0 i}, K_{1 i}$,
$K_{2 i}, K_{3 i}$ denote the 4 key bytes in the column $K_{i}$. Note that the signal $\mathrm{SEL}_{E D}$ is made 1 only during cycles 8 , $12,16,20$ of the decryption phase. The flow of data has been explained in Figure 3 .

It can be seen that at cycle 8 , the three rightmost key registers in the bottommost row have the key bytes $L_{00}, L_{01}, L_{02}$. At this point $\mathrm{SEL}_{E D}$ is set to 1 . Thus in the next cycle the bottommost key row would contain the bytes $L_{00}, K_{01}=L_{00} \oplus L_{01}, K_{02}=L_{01} \oplus$ $L_{02}, K_{03}=L_{02} \oplus L_{03}$ respectively. Similar additions occur at cycles 12,16 and 20 and as a result at the beginning of cycle 0 of the next round the four columns of the key register would have the values $L_{0}, K_{1}, K_{2}, K_{3}$ respectively. Thereafter in cycles 1 to $4, F\left(K_{3}\right)$ is computed in the same manner as described in the encryption cycles and added to $L_{0}$ in the first column. And as a result at the beginning of cycle 5, the key columns contain $K_{0}=L_{0} \oplus F\left(K_{3}\right), K_{1}, K_{2}, K_{3}$ which is the complete next roundkey. Since the complete roundkey is already available, the SELXOR signal controlling the xor gate in the topmost row is zeroed as the roundkeys are serially driven out for the add roundkey operation. Thus all the functionalities of Inverse Keyschedule are completely accommodated using this architecture. Furthermore the complete decryption roundkey is available from cycles 5 through 20, which is incidentally the period during which we perform the add roundkey operation.

\subsection{Sequence of operations}

Unlike ciphers like Midori 4, Prince [8] and Noekeon 13. AES was probably not concieved as an efficiently implementable involutive cipher. As a result, the sequence of operations during the encryption and decryption flow are quite different. The sequence of operation during the encryption flow is as follows:

1. Add whitening key.

2. Rounds 1 to 9

A. Substitution layer, B. Shiftrows,

C. Mixcolumn, D. Add roundkey

3. Round 10

A. Substitution layer, B. Shiftrows, 

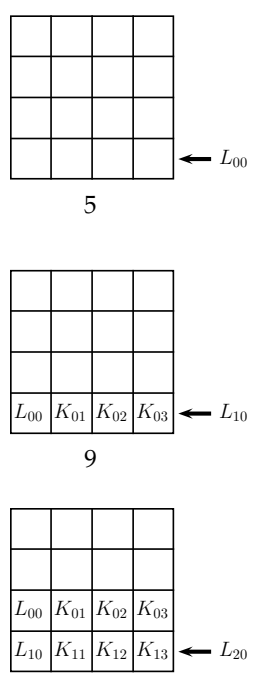

13

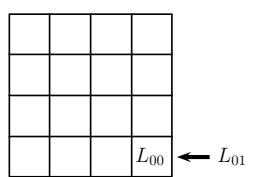

6

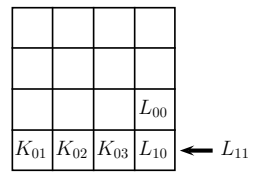

10

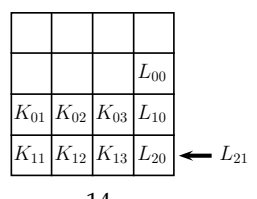

14

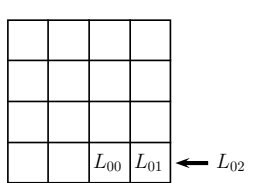

7

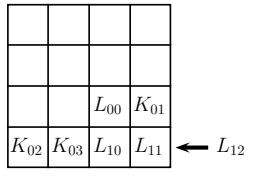

11

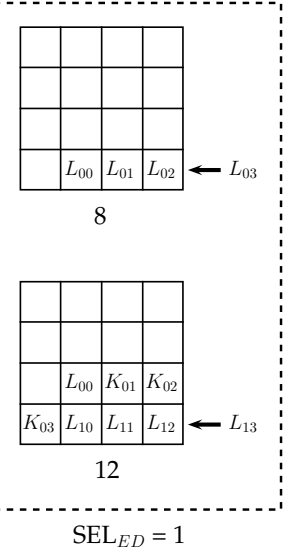

w.......

Fig. 3: Data flow in the Key registers during Decryption

\section{Add roundkey}

As previously mentioned, the 21 cycle encryption phase is arranged as Shiftrow $\rightarrow$ Mixcolumn $\rightarrow$ Add roundkey + Substitution layer of next round. The decryption flow of operations must exactly be opposite of encryption. Since the Shiftrows/Inverse Shiftrows can be commuted with S-box/Inverse S-box operation respectively, we can go with the following composition of one decryption round (also used in the architecture in [26]):

$$
\begin{aligned}
\text { Inv. Shiftrow } \rightarrow \text { Inv. Mixcolumn } \rightarrow & \text { Add roundkey } \\
& + \text { Inv. S-box }
\end{aligned}
$$

This sequence is attractive in this particular architecture because it has exactly the same order of operations as in encryption, and so it does not need too many changes in the underlying control system that produces select signals for the various multiplexers in the circuit. However as mentioned in 26, this sequence essentially swaps the order of Add roundkey and Inverse Mixcolumn operations. Since Mixcolumn and hence also Inverse Mixcolumn are linear functions, this requires the Inverse Mixcolumn function to be operated on the current roundkey before using it during the Add roundkey operation (since $M C^{-1}(X+K)=M C^{-1}(X)+$ $\left.M C^{-1}(K)\right)$. There are two ways to achieve this: a) use an additional circuit for Inverse Mixcolumns or b) spend extra cycles to compute the Inverse Mixcolumn of the current roundkey. Option a increases circuit size and option b increases latency.

In this paper we propose an alternate sequence of the decryption cycle that compromises on neither the circuit size nor latency. We propose the following flow:

$$
\begin{array}{r}
\text { Inv. Mixcolumn } \rightarrow \text { Inv. Shiftrow } \rightarrow \text { Inv. S-box }+ \\
\text { Add roundkey }
\end{array}
$$

Since this sequence of operations is essentially the mirror inverse of the AES encryption round function, no swapping of Add roundkey and Inverse Mixcolumn is needed, and that obviates the need to calculate the Inverse Mixcolumn of the roundkey. To better explain the operations, let us present a cycle by cycle breakdown of the 21 cycle decryption round function. The decryption starts with the addition of the whitening key. The finite state machine (FSM) generating the round signals is again initialized to cycle number 5 . So in cycles numbered 5 to 20 (i.e. the very first 16 cycles) the following transformations take place:

Cycles 5 to 20: The 8 bit chunks of ciphertext and key are respectively filtered out of the main state and key multiplexers respectively They are xored, and the resultant signal fed to the state registers. Note that in the corresponding encryption stage, we additionally calculated the S-box of the first round. Hence in order to accommodate both encryption and decryption we need a multiplexer after the Sbox circuit as shown in Figure 2 The Key bytes are input to key register " 33 ", from where it is serially forwarded in the next round. However as mentioned in the previous subsection, the $\mathrm{SEL}_{E D}$ signal is set to 1 at rounds 8, 12, 16, 20 due to which at beginning of the next phase, the Key four register columns hold the value $L_{0}, K_{1}, K_{2}, K_{3}$ respectively. 


ENCRYPTION
\begin{tabular}{|c|l|l|l|l|}
\hline \multirow{2}{*}{0} & State & & & Add Whitening Key + S-box of 1st round \\
\cline { 2 - 6 } & Key & & & Store Key serially \\
\hline \multirow{2}{*}{$1-10$} & State & Shiftrow & Mixcolumn & Add roundkey + S-box of next round \\
\cline { 2 - 5 } & Key & Frozen & Compute $F\left(K_{3}\right)$ & Compute roundkey + Store it serially \\
\hline
\end{tabular}

\begin{tabular}{|c|c|c|c|c|}
\hline \multirow{2}{*}{\multicolumn{2}{|c|}{$\begin{array}{l}\text { DECRYPTION } \\
\text { Round }\end{array}$}} & 0 & $1-4$ & \multirow[t]{2}{*}{$5-20$} \\
\hline & & & & \\
\hline 0 & \multirow{2}{*}{ Key } & & & Store Key serially (with $\mathrm{SEL}_{E D}=1$ at $8,12,16,20$ ) \\
\hline $1-10$ & & Frozen & Compute $F\left(K_{3}\right)$ & Store Key serially (with $\mathrm{SEL}_{E D}=1$ at $8,12,16,20$ ) \\
\hline 0 & \multirow{2}{*}{ State } & & & Add Whitening Key \\
\hline $1-10$ & & Mixcolumn $^{-1}$ & Shiftrow $^{-1}$ & Inverse S-box + Add roundkey \\
\hline & & $0-3$ & 4 & $5-20$ \\
\hline
\end{tabular}

Fig. 4: Operation sequences in the Encryption/Decryption stages

After this the cycle counter is automatically reset to 0 , and each 21 cycle round function is executed 10 times. Since the data flow in the key registers has already been explained in the previous subsection, we concentrate on the state register.

Cycles 0 to 3: These cycles perform the Inverse Mixcolumn operation on the state columns, in exactly the same way Forward Mixcolumn is executed in the encryption stage in cycles 1 to 4 . However only in the very first round the Inverse Mixcolumn operation is bypassed, as required in AES decryption.

Cycle 4: This cycle is reserved for the Inverse Shiftrow operation.

Cycles 5 to 20: The bytes of state are taken out from register " 00 " and input into the combined Forward and Inverse S-box circuit to compute the Inverse S-box operation. The output of the S-box is then xored with the current roundkey byte from the key register " 00 " and circulated serially back into the state registers via the register marked " 33 ". Note that the order of S-box and Add roundkey in the decryption phase is exactly the opposite as the encryption phase. As a result we employ two 8-bit xor gates, one before and one after the S-box circuit, for key addition in the encryption and decryption stages respectively. The xor gate inputs are controlled by and gates as shown in Figure 2, in order to bypass the addition operation as required.

In the tenth and final round, the decrypted plaintext is made available from cycles 5 through 20 after the Add roundkey operation. The above process is explained pictorially in Figure 4. We now describe some of the components used in the circuit.

\subsection{S-box}

Over the years, there has been substantial research into compact circuit implementations of the AES S-box 9 , 12, 24, 26, 30. Almost all of them use the underlying algebraic structure of the AES S-box, that essentially combines an affine transformation with an inverse computation over the AES finite field. However the architecture due to Canright [12] remains one of the smallest in terms of circuit size for the combined Forward and Inverse S-box, and thus this is the architecture we chose for the combined S-box/Inverse S-box circuit.

3.6 Combined Forward and Inverse Mixcolumn Circuit

In [26, the authors use the following decomposition of the Inverse Mixcolumn matrix to achieve an efficient 
implementation:

$\left[\begin{array}{ccccc}14 & 11 & 13 & 9 \\ 9 & 14 & 11 & 13 \\ 13 & 9 & 14 & 11 \\ 11 & 13 & 9 & 14\end{array}\right]=\left[\begin{array}{llll}2 & 3 & 1 & 1 \\ 1 & 2 & 3 & 1 \\ 1 & 1 & 2 & 3 \\ 3 & 1 & 1 & 2\end{array}\right]+\left[\begin{array}{llll}8 & 8 & 8 & 8 \\ 8 & 8 & 8 & 8 \\ 8 & 8 & 8 & 8 \\ 8 & 8 & 8 & 8\end{array}\right]+\left[\begin{array}{llll}4 & 0 & 4 & 0 \\ 0 & 4 & 0 & 4 \\ 4 & 0 & 4 & 0 \\ 0 & 4 & 0 & 4\end{array}\right]$

The xxtime (i.e. multiplication by 4) operation in AES finite field can be implemented in 5 xor gates as shown ( $b_{6} \oplus b_{7}$ is computed just once and the output is reused to construct the 5th LSB)

$$
\begin{array}{r}
\operatorname{xxtime}\left(b_{7}, b_{6}, \ldots, b_{0}\right) \mapsto \quad\left(b_{5}, b_{4}, b_{3} \oplus b_{7}, b_{2} \oplus b_{6} \oplus b_{7},\right. \\
\left.b_{1} \oplus b_{6}, b_{0} \oplus b_{7}, b_{6} \oplus b_{7}, b_{6}\right)
\end{array}
$$

Using this implementation of xxtime, the authors proposed a construction of Inverse Mixcolumns using 193 xor gates and a 32 bit multiplexer. However a more efficient implementation is due to Paulo Barreto 14, Section 4.1.3], which factorizes the Inverse Mixcolumn matrix as :

$\left[\begin{array}{cccc}14 & 11 & 13 & 9 \\ 9 & 14 & 11 & 13 \\ 13 & 9 & 14 & 11 \\ 11 & 13 & 9 & 14\end{array}\right]=\left[\begin{array}{llll}2 & 3 & 1 & 1 \\ 1 & 2 & 3 & 1 \\ 1 & 1 & 2 & 3 \\ 3 & 1 & 1 & 2\end{array}\right] \cdot\left[\begin{array}{llll}5 & 0 & 4 & 0 \\ 0 & 5 & 0 & 4 \\ 4 & 0 & 5 & 0 \\ 0 & 4 & 0 & 5\end{array}\right]$

To implement the above circuit, we simply premultiply the input column by the Circulant $(5,0,4,0)$ matrix as follows:

$y_{3}=\operatorname{xxtime}\left(x_{3} \oplus x_{1}\right) \oplus x_{3}, \quad y_{2}=\operatorname{xxtime}\left(x_{2} \oplus x_{0}\right) \oplus x_{2}$

$y_{1}=\operatorname{xxtime}\left(x_{3} \oplus x_{1}\right) \oplus x_{1}, \quad y_{0}=\operatorname{xxtime}\left(x_{2} \oplus x_{0}\right) \oplus x_{0}$

where $X=\left(x_{3}, x_{2}, x_{1}, x_{0}\right)$ and $Y=\left(y_{3}, y_{2}, y_{1}, y_{0}\right)$ are the input and output columns of the multiplication block. The multiplication block takes exactly 58 xor gates. Thereafter we choose either $X$ for Mixcolumns or $Y$ for Inverse Mixcolumns, and input the resultant to the AES Mixcolumn circuit, as shown in Figure 5. Since the Mixcolumn circuit can be efficiently implemented in 108 gates, the combined circuit takes $108+58=166$ xor gates and a 32 bit multiplexer which is more efficient than the construction in 26 .

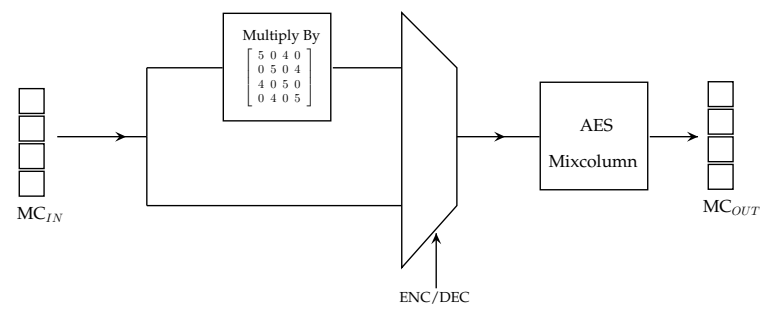

Fig. 5: Combined Forward and Inverse Mixcolumn circuit

\subsection{Round Constants and Control System}

We use LUT based round constants. If $r$ is the current round number, then the encryption operation uses LUT $(r)$, while the decryption operation uses LUT(11$r)$. The two signals can be input to an 8-bit multiplexer so that one can be chosen over the other as required. To further optimize, one can instead place a multiplexer before the LUT and choose between the 4-bit constants $r$ and $11-r$, and use the resultant signal as input to the LUT. Since this requires only a 4-bit multiplexer, it saves us additional area equivalent to a 4-bit multiplexer. Furthermore, all control signals are generated using a 21 cycle LFSR as described in [25].

\section{Atomic-AES v2.0: Architecture and Dataflow}

We will now present a full description of the proposed architecture for Atomic-AES v2.0 which provides dual functionalities for encryption and decryption. A diagram for the proposed architecture is presented in Figure 6. The Atomic-AES v2.0 architecture has strong structural similarities with the Atomic-AES described in the previous section, and thus it would be more expedient to highlight the salient dissimilarities between the two architectures before providing a complete description of the functionalities in the data and key path.

\subsection{Main changes}

There are two structural optimizations in the AtomicAES v2.0 architecture, due to which it was possible to reduce the area. They are listed as follows:

\section{Replacing scan flip-flops with ordinary flip-}

flops: One of the reasons why scan flip-flops are used for implementing both the state and key registers was that these storage units needed to support multiple modes of operation, in which each byte sized register needs to accept data from multiple sources. The state registers need to support serially loading and unloading data as well as the Shiftrow and Inverse Shiftrow operations. The key registers support 2 types of data movement: horizontal and vertical. the horizontal is meant for serial loading/unloading data, while the vertical is used to efficiently compute the nonlinear function $F$ used in the Keyschedule.

To begin, let us start with the Keyschedule. The vertical movement of data used to compute the $F$ function is required only in the outermost columns of the key registers, i.e. columns 0 and 3 . It is actually 


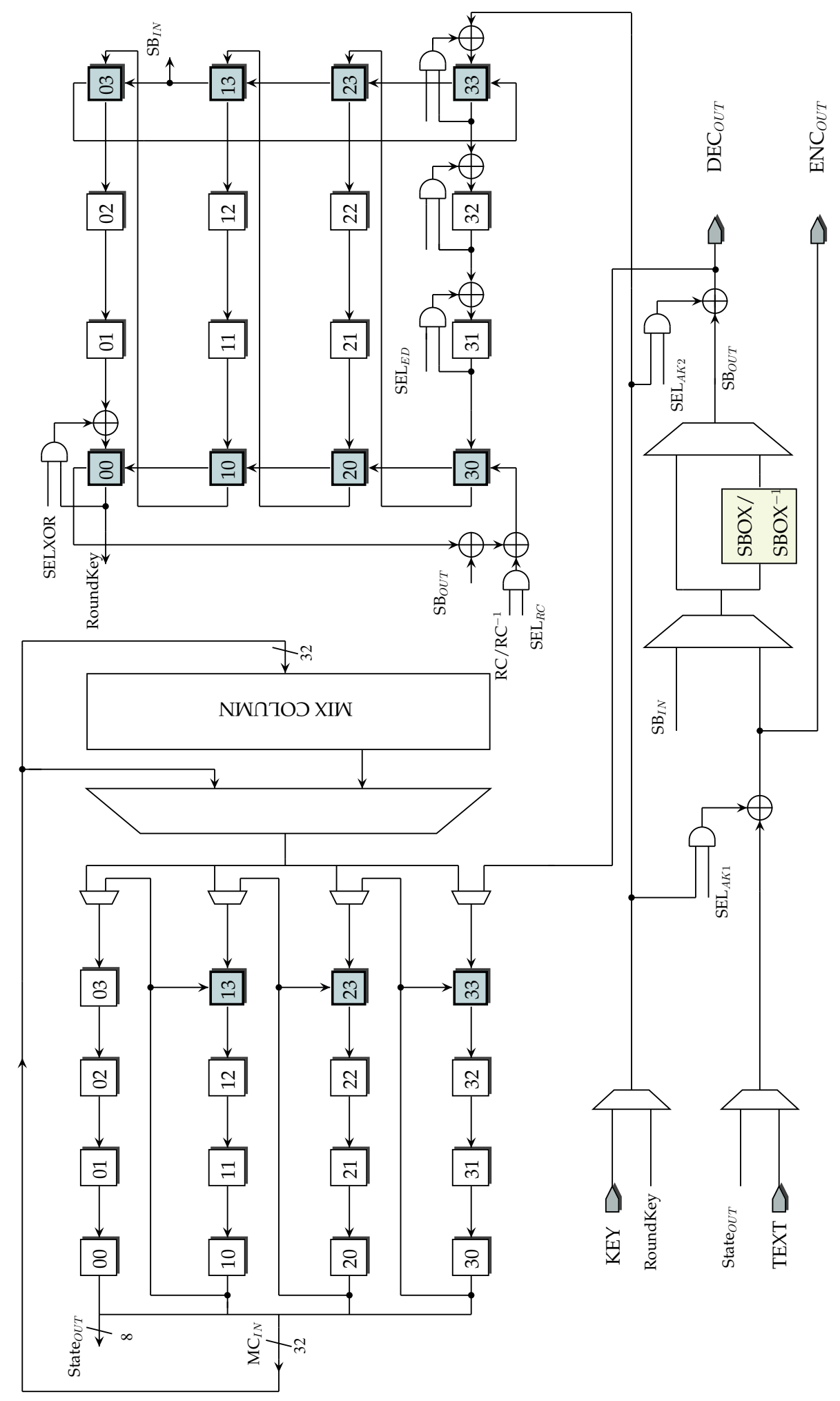

Fig. 6: The AES 8 bit Encryption/Decryption architecture for Atomic-AES v2.0 (boxes in grey denote byte registers made of scan flip-flops) 
not required in the two middle columns 1 and 2 . It is therefore possible to implement the middle-most columns with ordinary rather than scan flip-flops. Of course this requires that the data movement in the middle columns be frozen when the function $F$ is being calculated. This can be easily achieved using clock gating techniques.

In the state registers, we argue that scan flip-flops are required to implement only the byte registers "13", " $23 "$ and " 33 ". These are the byte registers in the final column of rows $1,2,3$ respectively. Scan flip-flops are not required for " 03 " because the zeroth row does not require data movement during the Shiftrow or Inverse Shiftrow operations. For either the Shiftrow or Inverse Shiftrow operations, there is a maximum movement of three columns to the left for any row. Indeed, except the zeroth row which does not require data movement, any row which has a movement of $x$ columns towards the left for Shiftrow would undergo a movement of $4-x$ columns towards the left for Inverse Shiftrows. Thus if the designer is prepared to allow 3 clock cycles for the Shiftrow/Inverse Shiftrow operation then both operations can be achieved by single directional data movement towards the left. This is precisely why, the remaining byte registers can be implemented with ordinary flip-flops. However, the designer has to take help of clock gating to freeze data movement in certain rows during the row-wise shifting operation. This has been tabulated in Table 2. As we will see shortly, during the Encryption flow, the Shiftrow is executed in cycles labelled 0, 1, 2 and during Decryption the Inverse Shiftrow operation is executed in cycles 12, 13, 14. Figure 6. gives a complete picture of the architecture. Registers implemented using scan flip-flops are labeled in grey. Except for 3 registers in the state and 8 in the key, all can be implemented using ordinary flip-flops. Since ordinary flip-flops occupy approximately 1 GE less than scan flip-flops, this saves us around $25 \times 8=200 \mathrm{GE}$. In addition, we do not need to use 2 extra 8-bit multiplexers used in the state registers in Atomic-AES. So the total savings is around 230 GE minus some additional logic used to implement the clock gating signals.

\section{Replacing Combined Mixcolumn circuit with} Forward Mixcolumn: In the previous section we were using a combined Mixcolumn/Inverse Mixcolumn circuit which took 166 xor gates and a 32 bit multiplexer. Since the circuit operated on a column every clock cycle, a total of 4 cycles were required to compute the Mixcolumn over the entire state. In this work, we take advantage of the fact that the Inverse Mixcolumn matrix used in AES is the cube of the Forward Mixcolumn matrix, i.e.

$\left[\begin{array}{cccc}14 & 11 & 13 & 9 \\ 9 & 14 & 11 & 13 \\ 13 & 9 & 14 & 11 \\ 11 & 13 & 9 & 14\end{array}\right]=\left[\begin{array}{llll}2 & 3 & 1 & 1 \\ 1 & 2 & 3 & 1 \\ 1 & 1 & 2 & 3 \\ 3 & 1 & 1 & 2\end{array}\right]^{3}$

This directly implies that if the designer runs the Forward Mixcolumn operation 3 times over the state i.e. for $3 \times 4=12$ cycles, he would functionally achieve the Inverse Mixcolumn operation. This in turn means that a Forward Mixcolumn circuit which occupies 108 xor gates is sufficient for both purposes. This saves us area equal to 58 xor gates and one 32 bit multiplexer, which amounts to around 130 GE. Note that in most standard cell libraries, this would ordinarily lead to a saving of over 200 GE, instead of just 130 GE. However, we need to realize that the Combined Mixcolumn circuit as shown in Figure 5 is only a simplistic representation of the logic blocks involved in the circuit. In practice, when the synthesizer optimizes the Combined Mixcolumn circuit for area, it is constructed using a network of and/xor gates which ends up taking area which is usually less than the sum of the areas of the Forward Mixcolumn circuit, 58 xor gates and a 32 bit multiplexer. Hence the area savings of the Forward Mixcolumn circuit over the combined circuit is only around $130 \mathrm{GE}$.

\subsection{Encryption Flow}

The encryption flow is almost the same as the one used in Atomic-AES and which has been described in brief in the previous section and it maintains exactly the same order of operations. There are subtle differences however. Since Shiftrow is executed over 3 cycles rather than 1 , one encryption round is carried out over 23 cycles rather than 21 . The circuit uses a maximum length 5 bit LFSR to generate control signals, which has a period of 31 cycles which we label as 0 to 30 . In the beginning the control system is initialized to cycle 15 .

Cycles 15 to 30: As before, the initial $S(P T \oplus K)$ operation is performed and the result is stored serially in the state registers and the key bytes are stored serially in the key registers.

Thereafter the counter is reset to 0 , and the 10 encryption rounds are executed one after the other. Each 


\begin{tabular}{c|c|c|c|c||c|c|c} 
& & \multicolumn{4}{|c||}{ Shiftrow Cycles } & \multicolumn{3}{c}{ Inverse Shiftrow Cycles } \\
\hline$\#$ & Row & 0 & 1 & 2 & 12 & 13 & 14 \\
\hline \hline 1 & $\mathbf{0}$ & $\mathrm{F}$ & $\mathrm{F}$ & $\mathrm{F}$ & $\mathrm{F}$ & $\mathrm{F}$ & $\mathrm{F}$ \\
\hline 2 & $\mathbf{1}$ & $\mathrm{F}$ & $\mathrm{F}$ & $\mathrm{O}$ & $\mathrm{O}$ & $\mathrm{O}$ & $\mathrm{O}$ \\
\hline 3 & $\mathbf{2}$ & $\mathrm{F}$ & $\mathrm{O}$ & $\mathrm{O}$ & $\mathrm{F}$ & $\mathrm{O}$ & $\mathrm{O}$ \\
\hline 4 & $\mathbf{3}$ & $\mathrm{O}$ & $\mathrm{O}$ & $\mathrm{O}$ & $\mathrm{F}$ & $\mathrm{F}$ & $\mathrm{O}$ \\
\hline
\end{tabular}

Table 2: Data flow in the rows of state registers during Shiftrow/Inverse Shiftrow. (F: Frozen, O: Operational)

\section{ENCRYPTION}

\begin{tabular}{|c|c|c|c|c|c|}
\hline & & $0-2$ & $3-6$ & $7-14$ & $15-30$ \\
\hline \multicolumn{6}{|c|}{ Round } \\
\hline \multirow{2}{*}{0} & State & & & \multirow{4}{*}{$\begin{array}{l}\text { Control System } \\
\text { jumps from } 6 \\
\text { to } 15 \text { during } \\
\text { ENCRYPTION }\end{array}$} & Add Whitening Key + S-box of 1st round \\
\hline & Key & & & & Store Key serially \\
\hline \multirow{2}{*}{$1-10$} & State & Shiftrow & Mixcolumn & & Add roundkey + S-box of next round \\
\hline & Key & Frozen & Compute $F$ & & Compute roundkey + Store it serially \\
\hline
\end{tabular}

\begin{tabular}{|c|c|c|c|c|c|c|}
\hline \multicolumn{7}{|c|}{ DECRYPTION } \\
\hline & & $0-2$ & $3-6$ & & 7-14 & $15-30$ \\
\hline \multicolumn{7}{|c|}{ Round } \\
\hline 0 & \multirow{2}{*}{ Key } & & & & & Store Key serially (with $\mathrm{SEL}_{E D}=1$ at $18,22,26,30$ ) \\
\hline $1-10$ & & Frozen & Compute $F$ & Frozen & & Store Key serially (with $\mathrm{SEL}_{E D}=1$ at $18,22,26,30$ ) \\
\hline 0 & \multirow{2}{*}{ State } & & & & & Add Whitening Key \\
\hline $1-10$ & & \multicolumn{3}{|c|}{ Mixcolumn $^{-1}$} & Shiftrow $^{-1}$ & Inverse S-box + Add roundkey \\
\hline & & \multicolumn{3}{|c|}{$0-11$} & $12-14$ & $15-30$ \\
\hline
\end{tabular}

Fig. 7: Operation sequences in the Encryption/Decryption stages

round consists of the following ordered sequence of operations:

Cycles 0 to 2: The state registers execute Shiftrow, and the Key registers are frozen.

Cycles 3 to 6: The state registers execute Mixcolumn, and the outermost columns of the Key register compute the function $F$ as explained in the previous section. During encryption, the control system transitions from cycle 6 to cycle 15 , so that cycles 7 to 14 do not occur.

Cycles 15 to 30: Exactly as in the previous section, the bytes are driven serially out of " 00 " from both the state and key side, the Add roundkey and Substitution layer of the next round are performed and the resultant signal, and the key bytes are driven serially back into the state/key registers respectively.

The encryption function thus takes $23 \times 10+16=246$ cycles to complete.

\subsection{Decryption Flow}

The decryption flow is also almost the same as the one used in Atomic-AES and exactly the same order of operations is maintained. The main differences are that Inverse Mixcolumn is executed over 12 cycles and Inverse Shiftrow over 3 cycles. Thus one decryption round takes 31 cycles to complete. As before, in the beginning the control system is initialized to cycle 15 .

Cycles 15 to 30: As before the initial whitening key addition i.e. $C T \oplus K$ operation is performed and the result is stored serially in the state registers and the key bytes are stored serially in the key registers. As explained in the previous section, the $S E L_{E D}$ signal is set to 1 in cycles $18,22,26,30$ to enable efficient backward generation of the roundkeys.

Thereafter the counter is reset to 0 , and the 10 decryption rounds are executed one after the other. Each round consists of the following ordered sequence of operations: 

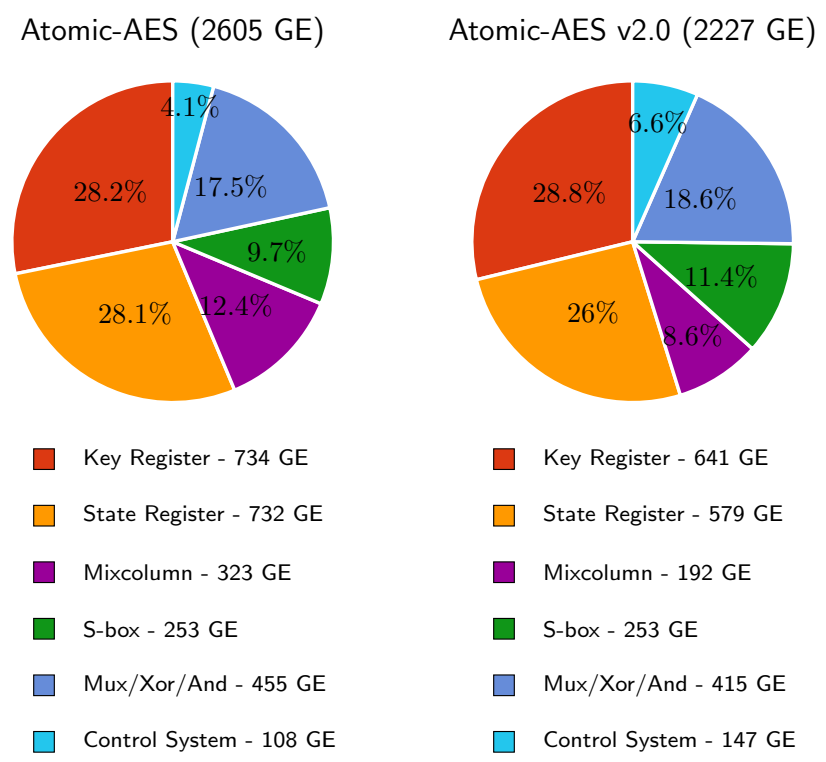

Fig. 8: Area requirements of the individual components (using the STM 90nm logic process )

Cycles 0 to 14: Now, the state registers execute Inverse Mixcolumn during 0 to 11, and then Inverse Shiftrow during 12 to 14 . The key registers are frozen during 0 to 2 and again from 7 to 14 . In the 4 cycles in between, (i.e. during 3 to 6 ) the non-linear function $F$ is computed exactly as explained in the previous section.

Cycles 15 to 30: The bytes are driven serially out of " 00 " from both the state and key side, the Inverse S-box is applied on the state bytes after which the add roundkey is performed and the resultant signal, and the key bytes are driven serially back into the state/key registers respectively. The $S E L_{E D}$ signal is again set to 1 in cycles $18,22,26,30$ to enable efficient backward generation of the next roundkey.

The decryption function thus takes $31 \times 10+16=326$ cycles to complete. The flow has also been explained diagrammatically in Fig 7 .

\subsection{Control System}

All control signals are generated using a maximal length 31 cycle LFSR. Some additional logic is used to sense the clock cycle 6 in the encryption cycle and transition to cycle 15 .

\section{Performance Evaluation}

In order to perform a fair performance evaluation, we implemented the circuit using VHDL. Thereafter the following design flow was adhered to for all the circuits: a functional verification at the RTL level was first done using Mentor Graphics Modelsim software. The designs were synthesized using the standard cell library of the following logic processes

1. STM $90 \mathrm{~nm}$ logic process(CORE90GPHVT v 2.1.a),

2. STM $65 \mathrm{~nm}$ logic process(CORE65LPLVT v 5.1),

3. UMC $90 \mathrm{~nm}$ low leakage logic process,

4. TSMC $90 \mathrm{~nm}$ logic process,

with the Synopsys Design Compiler, with the compiler being specifically instructed to optimize the circuit for area. A timing simulation was done on the synthesized netlist to confirm the correctness of the design, by comparing the output of the timing simulation with known test vectors. The switching activity of each gate of the circuit was collected while running post-synthesis simulation. The average power was obtained using Synopsys Power Compiler, using the back annotated switching activity. The results are tabulated in Table 3 .

We outline some of the essential lightweight metrics of known implementations of encryption/decryption architectures of AES and compare it with the two architectures proposed here. Energy consumption was listed rather than power as it is a measure of the total electrical work done during one encryption/decryption. Since the circuits in Table 3 are implemented using different CMOS logic processes, there are most likely to be wide variations in energy consumption and maximum throughput. For example the throughput of 23 is quite high as it is implemented using the standard cell library of the $22 \mathrm{~nm}$ CMOS logic process which is faster 


\begin{tabular}{|c|c|c|c|c|c|c|c|}
\hline \# & Architecture & Type & Library & $\begin{array}{l}\text { Area } \\
\text { (GE) }\end{array}$ & $\begin{array}{l}\text { Latency } \\
\text { (cycles) }\end{array}$ & $\begin{array}{c}\text { Energy } \\
(\mathrm{nJ})\end{array}$ & $\begin{array}{l}T P_{\max } \\
(\mathrm{Mbps})\end{array}$ \\
\hline 1 & 8-bit Serial [25] & $E$ & UMC $180 \mathrm{~nm}$ & 2400 & 226 & 8.4 & - \\
\hline 2 & Grain of Sand [17] & ED & Philips 350nm & 3400 & $1032 / 1165$ & $46.4 / 52.4$ & $9.9 / 8.8$ \\
\hline 3 & 8-bit Serial [23] & ED & $22 \mathrm{~nm}$ & 4037 & $336 / 216$ & $3.9 / 2.5$ & $432 / 671$ \\
\hline 4 & 32-bit Serial [26] & ED & $110 \mathrm{~nm}$ & 5400 & $54 / 54$ & - & 311 \\
\hline \multirow[t]{4}{*}{5} & \multirow[t]{4}{*}{ Atomic-AES } & \multirow[t]{4}{*}{ ED } & STM 90nm & 2605 & \multirow[t]{4}{*}{$226 / 226$} & 3.3 & 93.8 \\
\hline & & & STM 65nm & 2931 & & 2.2 & 58.4 \\
\hline & & & UMC 90nm & 3413 & & 3.2 & 82.2 \\
\hline & & & TSMC 90nm & 3007 & & 2.5 & 67.0 \\
\hline \multirow[t]{4}{*}{6} & \multirow[t]{4}{*}{ Atomic-AES v2.0 } & \multirow[t]{4}{*}{ ED } & STM 90nm & 2227 & \multirow[t]{4}{*}{$246 / 326$} & $3.2 / 4.3$ & $88.4 / 66.7$ \\
\hline & & & STM 65nm & 2678 & & $1.9 / 2.5$ & $54.4 / 41.1$ \\
\hline & & & UMC 90nm & 2700 & & $2.6 / 3.4$ & $79.3 / 59.9$ \\
\hline & & & TSMC 90nm & 2569 & & $2.2 / 2.9$ & $65.0 / 49.1$ \\
\hline
\end{tabular}

Table 3: Performance Comparison of Atomic-AES with previous architectures in literature (Figures separated by '/' indicate corresponding figures for encryption/decryption, E: Encryption only, ED: ENC/DEC)

than the other logic processes listed in the table. The throughput of [26] is also high as it is a 32-bit serial circuit and thus has considerably lower latency.

In Figure 8 , we present a componentwise breakdown of the circuit sizes of the two architectures. We use clock gating to generate the clock for the State/Key registers, since the data movement has to be frozen for a few clock cycles. For the Atomic-AES architecture, apart from the multiplexers included in the implementation of the combined Forward and Inverse S-box, Mixcolumn and Round Constants, a quick glance at Figure 2 . tells us that we need

1. Six 8-bit multiplexers around the state register, one 32-bit multiplexer to bypass the Mixcolumn circuit, one 8-bit multiplexer after the S-box, and two 8-bit multiplexers to filter the raw key/plaintext (ciphertext) and the roundkey/state byte respectively.

2. Apart from this six 8-bit xors around the key registers and two 8-bit xors during state-key addition.

3. One input of 7 out of the 8 xor gates is controlled by an and gate.

This adds up to around 455 GE for the multiplexers, xor, and gates in the circuit. The LSFR based control system and the round constants take around 108 GE. This leads to 2605 GE for the entire circuit.

For the Atomic-AES v2.0 architecture, the modifications introduced imply that we need the following overhead (see Figure 6)

1. Two multiplexers less around the State registers, because we use two extra cycles to perform Forward/Inverse Shiftrow.

2. Considerable savings in the implementation of the state and key registers (due to 21 byte size scan registers being replaced by ordinary flip-flops).

3. Savings due to Combined Mixcolumn circuit being replaced by a Forward Mixcolumn circuit.
4. Slightly more logic to design the LFSR based control system, Round Constants and timing signals.

This implies that we need 415 GE for the multiplexers, xor, and gates in the circuit. The LSFR based control system, the round constants and the logic for clockgating take around $147 \mathrm{GE}$. Adding up the componentwise area figures as shown in Figure 8 , this leads to $2227 \mathrm{GE}$ for the entire circuit.

\section{Conclusion}

In this work, we present two compact architectures for AES: Atomic-AES and Atomic-AES v2.0 that perform the dual function of encryption and decryption. The circuits can be synthesized using 2605 and 2227 GE area respectively, using the standard cell library of the STM 90nm CMOS logic process. While Atomic-AES uses 226 cycles for both encryption and decryption, Atomic-AES v2.0 has an encryption/decryption latency of 246/326 cycles respectively. This is a substantial improvement over the Grain of sand implementation that has an area of $3400 \mathrm{GE}$ but a latency of over 1000 cycles for both encryption and decryption.

\section{References}

1. F. Abed, S. Fluhrer, J. Foley, C. Forler, E. List, S. Lucks, D. Mcgrew, J. Wenzel. The POET Family of On-Line Authenticated Encryption Schemes. Submission to the CAESAR competition. Available at https: //competitions.cr.yp.to/round1/poetv101.pdf.

2. E. Andreeva, A. Bogdanov, A. Luykx, B. Mennink, E. Tischhauser, K. Yasuda. AES-COPA v.1. Submission to the Caesar Compedition. Available at http:// competitions.cr.yp.to/round1/aescopav1.pdf. 
3. S. Banik, A. Bogdanov, F. Regazzoni. Exploring Energy Efficiency of Lightweight Block Ciphers. In SAC 2015, LNCS, vol. 9566, pp. 178-194, 2015.

4. S. Banik, A. Bogdanov, T. Isobe, K. Shibutani, H. Hiwatari, T. Akishita, F. Regazzoni. Midori: A Block Cipher for Low Energy. In ASIACRYPT 2015, LNCS, vol. 9453, pp. 411-436, 2015.

5. S. Banik, A. Bogdanov, F. Regazzoni, T. Isobe, H. Hiwatari, T. Akishita. Round gating for low energy block ciphers. In IEEE Hardware Oriented Security and Trust (HOST), pp. 55-60, 2016.

6. R. Beaulieu, D. Shors, J. Smith, S. Treatman-Clark, B. Weeks, L. Wingers. The Simon and Speck Families of Lightweight Block Ciphers. In IACR eprint archive. Available at https://eprint.iacr.org/2013/404.pdf.

7. A. Bogdanov, L. Knudsen, G. Leander, C. Paar, A. Poschmann, M. Robshaw, Y. Seurin, C. Vikkelsoe. PRESENT: An Ultra-Lightweight Block Cipher. In CHES 2007, LNCS, vol. 4727, pp. 450-466, 2007.

8. J. Borghoff, A. Canteaut, T. Güneysu, E. B. Kavun, M. Knežević, L. R. Knudsen, G. Leander, V. Nikov, C. Paar, C. Rechberger, P. Rombouts, S. S. Thomsen, T. Yalçin. PRINCE - A Low-Latency Block Cipher for Pervasive Computing Applications - Extended Abstract. In Asiacrypt 2012, LNCS, vol. 7658, pages 208-225, 2012.

9. J. Boyar, P. Matthews, R. Peralta. Logic Minimization Techniques with Applications to Cryptology. In J. Cryptology, vol. 26, pp. 28-312, 2013.

10. P. Chodowiec, K. Gaj. Very Compact FPGA Implementation of the AES Algorithm. In CHES 2003, LNCS, vol. 2779, pp. 319-333, 2003.

11. C. De Cannière, O. Dunkelman, M. Knežević. KATAN and KTANTAN - a family of small and efficient hardware-oriented block ciphers. In CHES 2009, LNCS, vol. 5747, pp. 272-288, 2009.

12. D. Canright. A very compact S-Box for AES. In CHES 2005, LNCS, vol. 3659, pp. 441-455, 2005.

13. J. Daemen, M. Peeters, G. V. Assche, V. Rijmen. Nessie Proposal: NOEKEON. Available at http://gro. noekeon.org/Noekeon-spec.pdf

14. J. Daemen, V. Rijmen. The design of Rijndael: AES - the Advanced Encryption Standard. Springer-Verlag, 2002.

15. N. Datta and M. Nandi. ELmD v1.0. Submission to the Caesar compedition. Available at https:// competitions.cr.yp.to/round1/elmdv10.pdf.

16. M. Dworkin. Recommendation for Block Cipher Modes of Operation. NIST Special Publication 800-38A. Available at http://csrc.nist.gov/publications/nistpubs/ 800-38a/sp800-38a.pdf.

17. M. Feldhofer, J. Wolkerstorfer, V. Rijmen. AES Implementation on a Grain of Sand. In IEEE Proceedings of Information Security, vol. 152(1), pages 13-20, 2005.

18. Z. Gong, S. Nikova, Y.W. Law. KLEIN: a new family of lightweight block ciphers. In RFIDSec 2011, LNCS, vol. 7055, pp. 1-18, 2011.
19. J. Guo, T. Peyrin, A. Poschmann, M. J. B. Robshaw. The LED Block Cipher. In CHES 2011, LNCS, vol. 6917, pp. 326-341, 2011.

20. P. Hämäläinen, T. Alho, M. Hännikäinen, and T. D. Hämäläinen. Design and Implementation of Low-Area and Low-Power AES Encryption Hardware Core. In DSD, pages 577-583, 2006.

21. D. Hong, J. Sung, S. Hong, J. Lim, S. Lee, B. Ko, C. Lee, D. Chang, J. Lee, K. Jeong, H. Kim, J. Kim, S. Chee. HIGHT: A New Block Cipher Suitable for Low-Resource Device. In CHES 2006, LNCS, vol. 4249, pp. 46-59, 2006.

22. A. Lutz, J. Treichler, F. Gürkaynak, H. Kaeslin, G. Basler, A. Erni, S. Reichmuth, P. Rommens, S. Oetiker, W. Fichtner. 2Gbit/s hardware realizations of RIJNDAEL and SERPENT: A comparative analysis. In CHES 2002, LNCS, vol. 2523, pp. 144158, 2002.

23. S. Mathew, S. Satpathy, V. Suresh, M. Anders, H. Kaul, A. Agarwal, S. Hsu, G. Chen, R.K. Krishnamurthy. $340 \mathrm{mV}-1.1 \mathrm{~V}, 289 \mathrm{Gbps} / \mathrm{W}, 2090$-gate nanoAES hardware accelerator with area-optimized encrypt/decrypt $\mathrm{GF}\left(2^{4}\right)^{2}$ polynomials in $22 \mathrm{~nm}$ tri-gate CMOS. In IEEE Journal of Solid-State Circuits, vol. 50, pp. 1048-1058, 2015.

24. N. Mentens, L. Batina, B. Preneel and I. Verbauwhede. A Systematic Evaluation of Compact Hardware Implementations for the Rijndael S-Box. In CT-RSA 2005, LNCS, vol. 3376, pp. 323-333, 2005.

25. A. Moradi, A. Poschmann, S. Ling, C. Paar, H. Wang. Pushing the Limits: A Very Compact and a Threshold Implementation of AES. In Eurocrypt 2011, LNCS, vol. 6632, pp. 69-88, 2011.

26. A. Satoh, S. Morioka, K. Takano, S. Munetoh. A Compact Rijndael Hardware Architecture with S-Box Optimization. In Asiacrypt 2001, LNCS, vol. 2248, pp. 239254, 2001.

27. K. Shibutani, T. Isobe, H. Hiwatari, A. Mitsuda, T. Akishita, T. Shirai. Piccolo: An Ultra-Lightweight Blockcipher. In CHES 2011, LNCS, vol. 6917, pp. 342-357, 2011.

28. T. Suzaki, K. Minematsu, S. Morioka, E. Kobayashi. TWINE: A Lightweight Block Cipher for Multiple Platforms. In SAC 2012, LNCS, vol. 7707, pp. 339-354, 2012.

29. R. Ueno, S. Morioka, N. Homma, T. Aoki. A High Throughput/Gate AES Hardware Architecture by Compressing Encryption and Decryption Datapaths - Toward Efficient CBC-Mode Implementation. In CHES 2016 , LNCS, vol. 9813, pp. 538-558, 2016.

30. R. Ueno, N. Homma, Y. Sugawara, Y. Nogami, and T. Aoki. Highly Efficient $\mathrm{GF}\left(2^{8}\right)$ Inversion Circuit Based on Redundant GF Arithmetic and Its Application to AES Design. In CHES 2015, LNCS, vol. 9293, pp. 63-80, 2015. 